\title{
Bupivacaína 0,25\% peridural, en la analgesia de parto; efectos hemodinámicos en la madre y feto
}

\author{
Silvia Arias $^{1}$, Celina Montes ${ }^{2}$
}

Resumen Objetivo: Valorar la bupivacaína 0,25\% por catéter epidural en el alivio del dolor de parto y efectos en la hemodinamia materna, progreso del parto y $\mathrm{pH}$ neonatal. Diseño: Estudio descriptivo, longitudinal, prospectivo. Lugar: Centro Obstétrico del Instituto Especializado Materno Perinatal, Lima, Perú, hospital de enseñanza universitaria. Pacientes: Sesenta parturientas en fase activa de trabajo de parto. Intervenciones: Se administró a las parturientas bupivacaína 0,25\% $12 \mathrm{~mL}$, vía catéter epidural, haciendo control del dolor con la escala visual análoga. Se registró la hemodinamia materno-fetal, pre y postanalgesia, dosis suplementaria de bupivacaína 0,25\% $6 \mathrm{~mL}$, trazado cardiotocográfico fetal, complicaciones. Se utilizó el programa estadístico SPSS 13,0. Principales medidas de resultados: Disminución del dolor, hemodinamia materna, $p H$ neonatal. Resultados: La administración de bupivacaína 0,25\% disminuyó significativamente el dolor ( $p<0,001$, prueba de Friedman), requiriendo 33 parturientas analgesia suplementaria (45 mg BPV prom.). La frecuencia basal fetal preanalgesia fue 142 lat/miny postanalgesia, 136 lat/min ( tstudentp < 0,05); el dermatoma alcanzado fue T8, con duración promedio de $115 \mathrm{~min}$; la satisfacción fue excelente en $50,1 \%$. Se usó oxitocina en $88,3 \%(13,1 \mathrm{mU} / \mathrm{min}, \mathrm{DE} 3,2 \mathrm{mU} / \mathrm{min})$, asociándose al acortamiento del tiempo de la fase activa en 2,42 hen primigestas y 1,55 hen multigestas; la cesárea fue necesaria en $11,7 \%$, el PH neonatal promedio 7,24. Conclusiones: La analgesia epidural con bupivacaína 0,25\% vía catéter es eficaz. La hemodinamia materna se mantiene, el latido cardiaco fetal desciende 12 latidos/min en promedio, la fase activa se acorta, estando relacionado al requerimiento de oxitocina.

Palabras clave Analgesia epidural; analgesia obstétrica; bupivacaína; concentración de iones de hidrógeno.

Peridural $0,25 \%$ bupivacaine analgesia for labor pain relief; effects on mother and fetus hemodynamics

\begin{abstract}
Objectives: To determine peridural bupivacaine analgesia for labor pain reliefand its effect on maternal and fetal hemodynamics. Design: Prospective, longitudinal, descriptive study. Setting: Obstetrical Center, Maternal and Perinatal Specialized Institute, Lima, Peru, a teaching hospital. Patients: Sixty patients in active phase of labor. Interventions: Twelve $m L$ of 0,25\% bupivacaine was given to the women in labor through epidural catheter. Visual analog scale was used to evaluate pain during labor. Maternal and fetal hemodynamic changes were described before and following epidural analgesia. Supplementary dosis of $6 \mathrm{~mL} 0,25 \%$ bupivacaine was used. Cardiotocographic tracings were registered and maternal and fetal complications were determined. Main outcome measures: Labor pain relief, maternal hemodynamics,
\end{abstract}

MRII Sub Especialidad Anestesiología Obstétrica, Instituto Nacional Materno Perinatal. Lima, Perú.

2 Médico Asistente Servicio de Anestesiología, Instituto Nacional Materno Perinatal. Lima, Perú. fetal $p H$. Results: There was statistically significant pain relief using $12 \mathrm{~mL}$ of $0,25 \%$ bupivacaine $(p<0,001)$. Supplementary dosis of bupivacaine was needed in 33 patients $(\bar{X}: 45 \mathrm{mg})$. Baseline fetal heart rate before and after epidural analgesia was 142 beats/ min and 136 beats/min, respectively $(p<0,05)$. Analgesic blockade reached T8; 115 minutes was the analgesia median time. Patient satisfaction was excellent in $50,1 \%$. Oxytocin was used in $88,3 \%$ patients (13,3 mIU/min $\pm 3,2 \mathrm{mIU} / \mathrm{min})$. Active phase labor length was shortened by 2,42 hours in primiparous and 1,55 hours in multiparous. Cesarean deliveries were peformed in 11,7\%. Mean neonatal pH was 7,24. Conclusions: Epidural analgesia with bupivacaine 0,25\% via catheter results in effective labor pain relief. Fetal heart beat decreased 12 beats/min. Phase active was shortened since oxytocin was used during this period.

Key words: Analgesia, epidural; analgesia, obstetrical; bupivacaine; hydrogen-ion concentration.

\section{INTRODUCCIÓN}

La analgesia epidural es la técnica utilizada con mayor frecuencia para el alivio del 
dolor, durante el trabajo de parto. Y es la forma más efectiva de analgesia de parto científicamente comprobada ${ }^{1}$ ). Un estudio aleatorizado controlado concluyó que la analgesia epidural con dosis bajas disminuye el porcentaje de partos vaginales instrumentales, sin comprometer la calidad de la analgesia $\left({ }^{2}\right)$.

Los objetivos de la analgesia regional en el trabajo de parto son claros: una analgesia eficaz con mínimos efectos adversos para la madre y el recién nacido $\left(^{3}\right)$. Lo correcto sería proveer alivio de parto con soluciones diluidas de anestésico local y corregir a tiempo las variaciones indeseadas del trazado cardiotocográfico fetal intraparto. Las nuevas formas de analgesia epidural utilizan combinaciones de opioides y menos anestésicos locales concentrados, que preservan la función motora materna; así como reducen la dosis del anestésico global, disminuyendo la incidencia e intensidad de los fenómenos cardiovasculares indeseables $\left({ }^{4-6}\right)$.

Los opioides administrados epiduralmente ganan acceso a los receptores opioides centrales en el cordón espinal su interacción con estos receptores produce analgesia sin bloqueo del nervio motor $\left({ }^{2}\right)$; su uso es restringido por el riesgo de cubrir receptores cefálicos y alcanzar los receptores opioides en el cerebro y que produzcan depresión respiratoria profunda e imprevisible y otros efectos como náusea, vómitos, prurito $\left({ }^{2}\right)$. Si bien estas técnicas están asociadas con mayor grado de satisfacción materna, los efectos sobre los resultados obstétricos son poco conocidos.

En una revisión de un artículo original se relacionó la analgesia epidural y efectos adversos en el neonato, distocias del parto e incremento de cesáreas, y sugirió informar a los padres acerca de la analgesia epidural asociada a distocia y fiebre maternal en nulíparas que experimentan prolongada labor con repetidos exámenes cervicales y ruptura prolongada de membranas que dejan infección clínica continuada de sepsis neonatal $\left({ }^{7}\right)$. Un estudio histológico de la placenta permitió comprobar que la fiebre no se presentaba como complicación de la analgesia epidural si no como consecuencia de un largo trabajo de parto que predisponía a infección (inflamación placentaria) ${ }^{8}$ ).

Una vieja controversia, dentro de la anestesia obstétrica, representa la potencial relación causal entre la aplicación de la analgesia epidural y la necesidad de realización de cesárea. Sin embargo, no parece ser el único factor determinante, sino que interactúa con otras variables, como la edad y la condición socioeconómica de la paciente $\left({ }^{9}\right)$.

En un estudio comparativo, se concluyó que la analgesia epidural no afecta los índices de cesáreas. Sin embargo, incrementa la utilización de oxitocina, la duración del segundo estadio del trabajo de parto y la instrumentación del parto vaginal $\left({ }^{9}\right)$.

En un estudio retrospectivo, aplicado a 126 gestantes en trabajo de parto sometidas a analgesia epidural, se encontró que los partos vaginales instrumentales fueron más frecuentes, $34,6 \%$; la cesárea fue significativamente más frecuente $-26 \%$ - que en la población control; recibieron sedación, resaltando que la duración de la fase activa fue de $231 \mathrm{~min}$ y el expulsivo 50 minutos, respectivamente $\left({ }^{10}\right)$.

\section{MATERIALES Y MÉTODOS}

Se realiza un estudio descriptivo, prospectivo, longitudinal, en el Centro Obstétrico del Instituto Especializado Materno Perinatal, durante los meses de octubre de 2005 a marzo de 2006. Se evaluó 60 parturientas, entre 13 y 40 años, previo consentimiento informado y siendo los criterios de inclusión inicio de trabajo espontáneo, gestación única, cefálico; dilatación cervical 4 $\mathrm{cm}$. Los pacientes fueron monitorizados con oximetría de pulso, monitorización cardioto- 
cográfico externa y presión arterial; se hidrató con 500 a $1000 \mathrm{~mL}$ de cloruro de sodio y, vía catéter epidural, se aplicó 12 $\mathrm{mL}$ de bupivacaina al $0,25 \%$.

El dolor fue controlado por EVA (escala visual análoga); considerándose 1 a 2 no dolor, 3 a 4 leve; 5 a 6 moderado; 7 a 8 severo; 9 a 10 muy severo. Se valoró estos datos en la preanalgesia y a $\operatorname{los} 15,30,60$, 90 y 120 minutos, respectivamente. El nivel sensitivo que alcanza determinado dermatoma; según la pérdida de sensibilidad al pinchazo (pin-pricktest) y el grado del bloqueo motor según la escala de Bromage $(0=$ puede levantar las extremidades inferiores; $1=$ puede flexionar rodillas y tobillos; $2=$ no puede flexionar rodillas y tobillos; $3=$ no puede flexionar tobillos), se continuó con la infusión de solución salina con o sin oxitocina, de acuerdo a las indicaciones ginecoobstétricas.

Se administró analgesia suplementaria (bupivacaína al $0,25 \%, 6 \mathrm{~mL}(15 \mathrm{mg})$ ), se anotó el Ápgar del recién nacido al primer y quinto minuto tras el nacimiento, se tomó muestra de sangre venosa de cordón umbilical para medir los gases arteriales antes de su clampaje, y el grado de satisfacción materna; en el puerperio, se registró las complicaciones del procedimiento epidural.

Las variables cuantitativas son presentadas como promedio, desviación estándar. Para evaluar la distribución de las variables cualitativas, se aplicó la prueba chi cuadrado. La comparación de variables cuantitativas se evaluó mediante la prueba $\mathrm{T}$ de student (se consideró significativo los valores $p<0,05)$, y para la evolución de la percepción del dolor se aplicó la prueba de Friedman.

\section{RESULTADOS}

Participaron 60 pacientes, con edades comprendidas entre 13 y 39 años, con un promedio de 24 años y una desviación estándar de 6,14; las gestantes adolescentes representaron $31 \%$ (19 casos).

En relación a las características generales, el nivel de estudios más frecuente fue la secundaria, en $75 \%$ (45 casos). $61,7 \%$ (37 pacientes) tuvo 6 a más controles prenatales, pero solo participó en talleres de psicoprofilaxis obstétrica $11,7 \%$ del total de pacientes.

El peso materno varió entre 54 y $80 \mathrm{~kg}$, con un promedio de $66,5 \mathrm{~kg}$. La edad gestacional promedio fue 39,3 semanas, $71,7 \%$ con edad gestacional entre 38 y 40 semanas; $61,7 \%$ (37 gestantes) fue primigesta y $11,7 \%$ multigesta ( 7 casos). Entre las condiciones médicas asociadas, podemos referir la anemia en 21,7\% (13 casos). La entidad obstétrica asociada al embarazo fue la rotura prematura de membranas, presente en $30 \%$ (18 casos) (Tabla 1).

El promedio de dilatación cervical fue de $5,25 \mathrm{~cm}$, la altura de presentación, -2 en $66,7 \%$ de los casos; la variedad de presentación más frecuente fue OIIA (45\%) y OIIT $(30 \%)$. La incorporación cervical fue $90 \%$ en $50 \%$ de las gestantes. El líquido amniótico fue de apariencia clara en 93,3\% (Tabla 2).

Todas las pacientes ingresaron con una presión arterial media en el rango normal, que descendió levemente a los 20 minutos postanalgesia, aun cuando estaban prehidratadas. La frecuencia cardiaca promedio mostró un leve descenso postanalgesia, cambiando de 80,8 a 79,0 ; de manera contraria, la saturación de oxígeno promedio aumentó de 95,0 a 96,3, por la aplicación de $4 \mathrm{~L} / \mathrm{min}$ de oxígeno humidificado (Tabla 3). la evaluación del dolor se realizó con la escala visual análoga, observándose una disminución progresiva y significativa $(p<0,001$, prueba de Friedman) del efecto de la analgesia con la dosis única, como aparece en la Tabla 4, Figura 1. 
Tabla 1. Características de las pacientes.

\begin{tabular}{|c|c|c|}
\hline & $\mathrm{n}$ & $\%$ \\
\hline \multicolumn{3}{|l|}{ Grupo etáreo (años) } \\
\hline Adolescentes & 19 & 31,0 \\
\hline En edad fértil & 37 & 63,0 \\
\hline Añosas & 4 & 6,0 \\
\hline \multicolumn{3}{|l|}{ Nivel de estudios } \\
\hline Primaria & 6 & 10,0 \\
\hline Secundaria & 45 & 75,0 \\
\hline Superior & 9 & 15,0 \\
\hline \multicolumn{3}{|l|}{ Estado civil } \\
\hline Soltera & 7 & 11,7 \\
\hline Casada & 17 & 28,3 \\
\hline Unión estable & 36 & 60,0 \\
\hline \multicolumn{3}{|l|}{ Controles prenatales } \\
\hline Sin control & 6 & 10,0 \\
\hline $1-5$ & 17 & 28,3 \\
\hline$\geq 6$ & 37 & 61,7 \\
\hline \multicolumn{3}{|l|}{ Psicoprofilaxis obstétrica } \\
\hline $\mathrm{Si}$ & 7 & 11,7 \\
\hline No & 53 & 88,3 \\
\hline \multicolumn{3}{|l|}{ Distribución según peso materno (kg) } \\
\hline $54-65$ & 27 & 45,0 \\
\hline $66-75$ & 29 & 48,3 \\
\hline $76-80$ & 4 & 6,7 \\
\hline \multicolumn{3}{|l|}{ Edad gestacional } \\
\hline$\leq 37$ & 4 & 6,6 \\
\hline$\geq 38 \leq 40$ & 43 & 71,7 \\
\hline$\geq 41$ & 13 & 21,7 \\
\hline \multicolumn{3}{|l|}{ Paridad } \\
\hline Primigestas & 37 & 61,7 \\
\hline Multigestas & 7 & 11,7 \\
\hline \multicolumn{3}{|l|}{ Condiciones médicas asociadas } \\
\hline Ninguno & 46 & 76,7 \\
\hline Anemia & 13 & 21,7 \\
\hline Diabetes mellitus & 1 & 1,6 \\
\hline \multicolumn{3}{|l|}{ Entidades obstétricas asociadas } \\
\hline Preeclampsia leve & 6 & 10 \\
\hline Rotura prematura de membranas & 18 & 30 \\
\hline Cesareada anterior $1 \mathrm{vez}$ & 3 & 5 \\
\hline Total & 60 & 100,0 \\
\hline
\end{tabular}

Recibieron $30 \mathrm{mg}$ de bupivacaina 22 pacientes, como dosis única; requiriendo una vez analgesia suplementaria 33 parturientas (45 mg BPV) y por segunda vez 5 pacientes (60 mg BPV); aún así, dos pacien-
Tabla 2. Características obstétricas antes del requerimiento de analgesia.

\begin{tabular}{lrr}
\hline & $\mathrm{n}$ & $\%$ \\
\hline Membranas & & \\
$\quad$ Íntegras & 38 & 63,3 \\
$\quad$ Rotas & 22 & 36,7 \\
Dilatación cervical (cm) & & \\
4 & 12 & 20,0 \\
5 & 21 & 35,0 \\
6 & 27 & 45,0 \\
Altura de presentación (cm) & & \\
-1 & 14 & 23,3 \\
-2 & 40 & 66,7 \\
-3 & 6 & 10,0 \\
Variedad de presentación & & \\
OIDP & 15 & 25,0 \\
OIIT & 18 & 30,0 \\
OIIA & 27 & 45,0 \\
Incorporación cervical & & \\
$80 \%$ & 6 & 10,0 \\
90\% & 50 & 83,3 \\
100\% & 4 & 6,7 \\
Características del líquido amniótico & & \\
Claro & 56 & 93,3 \\
Meconial fluido & 4 & 6,7 \\
Total & 60 & 100,0 \\
& & \\
\hline
\end{tabular}

tes experimentaron sutura dolorosa de episorrafia. No se observó bloqueo motor, presentando todos los pacientes una escala de Bromage igual a cero, permitiéndoles la movilidad de sus miembros inferiores y caderas. Respecto al nivel sensitivo, la mayoría alcanzó el dermatoma T8 (47,2\%),

Tabla 3. Cambios hemodinámicos maternos.

\begin{tabular}{lcr}
\hline & Promedio & DE \\
\hline Presión arterial media pre analgesia & 85,9 & 9,4 \\
Presión arterial media post analgesia & 79,1 & 8,3 \\
FC pre analgesia & 80,8 & 6,83 \\
FC postanalgesia & 79,0 & 6,56 \\
Saturación de oxígeno pre analgesia & 95,0 & 0,70 \\
Saturación de oxígeno post analgesia & 96,3 & 0,67 \\
\hline
\end{tabular}


Tabla 4. Resultado del promedio de la evaluación del dolor mediante la EVA según el tiempo de la evaluación ( $\mathrm{n}$ = 53).

\begin{tabular}{lccccc}
\hline & Pre analgesia & $\begin{array}{c}\text { Minuto } \\
15\end{array}$ & $\begin{array}{c}\text { Minuto } \\
30\end{array}$ & $\begin{array}{c}\text { Minuto } \\
60\end{array}$ & $\begin{array}{c}\text { Minuto } \\
90\end{array}$ \\
\hline Promedio & 8,7 & 5,5 & 2,7 & 2,3 & 1,4 \\
DE & 0,66 & 0,50 & 0,95 & 1,34 & 0,50 \\
\hline
\end{tabular}

$* p<0,001$ Prueba de Friedman.

T10 (34,0\%) y T6 (18,9\%). El nivel de satisfacción obtenido fue excelente, en 27 $(50,9 \%)$ de las pacientes, satisfacción buena en $24(45,3 \%)$ y presentaron alivio no satisfactorio $2(3,8 \%)$

La aplicación de oxitocina se requirió en $88,3 \%$ de los casos y el promedio requerido por estas paciente fue $13,1 \mathrm{mU} / \mathrm{min}$, con una DE de 3,2 $\mathrm{mU} / \mathrm{min}$; en $11,7 \%(\mathrm{n}=7)$ no hubo necesidad de oxitócicos (Tabla 5). La duración de la fase activa y la fase expulsiva, como se observa en esta tabla, es estadísticamente ( $p<0,05, \mathrm{~T}$ de student) mayor en primíparas que en multíparas. La vía de parto fue vaginal en $88,3 \% \quad(n=53)$ y los casos intervenidos por cesárea fueron por sufrimiento fetal agudo $(n=3)$.

En el trazado del monitoreo electrónico fetal intraparto, la línea de base promedio antes de la analgesia fue $142 \mathrm{lat} / \mathrm{min}$ (DE $7,4) \mathrm{y}$, posterior a la analgesia, 130 lat/

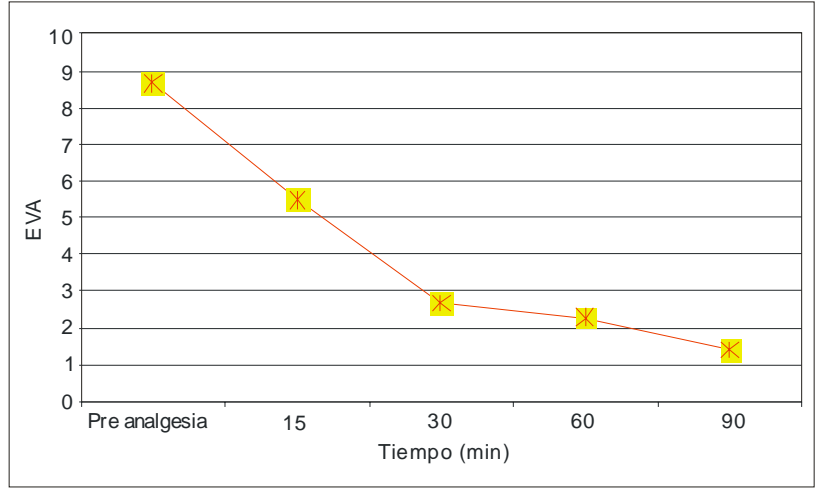

Figura 1. Estimación del dolor mediante la EVA según el tiempo de la evaluación ( $\mathrm{n}=53)$.
Tabla 5. Efectos primarios y secundarios.

\begin{tabular}{lrr}
\hline & $\mathrm{n}$ & $\%$ \\
\hline Vía de parto & & \\
$\quad$ Abdominal & 7 & 11,7 \\
$\quad$ Vaginal & 53 & 88,3 \\
Motivos de la cesárea & & \\
$\quad$ Ninguna & 53 & 88,3 \\
$\quad$ Sufrimiento fetal agudo & 3 & 5,0 \\
$\quad$ Mala actitud de presentación & 2 & 3,3 \\
$\quad$ Trabajo de parto disfuncional & 2 & 3,3 \\
& & \\
Dosis máxima de oxitocina (mU/min) postanalgesia & \\
$\quad$ No requirieron & 7 & 11,7 \\
8 & 9 & 15,0 \\
12 & 18 & 30,0 \\
16 & 24 & 40,0 \\
20 & 2 & 3,3
\end{tabular}

Duración de la fase activa en:*

$\begin{array}{lcc} & \text { Promedio (h:min) } & \text { DE (h:min) } \\ \text { Primigesta }(\mathrm{n}=33) & 2: 42 & 1,16 \\ \text { Multígesta }(\mathrm{n}=20) & 1: 55 & 0,46\end{array}$

Duración del período expulsivo en:**

\begin{tabular}{lcc} 
& Promedio $(\min )$ & DE $(\min )$ \\
Primíparas $(\mathrm{n}=33)$ & 28,5 & 7,5 \\
Multíparas $(\mathrm{n}=20)$ & 17,9 & 5,6 \\
\hline
\end{tabular}

* $p=0,017, t$ de student, ${ }^{* *} p<0,001$ t de student

min (DE 17,4), con una prueba $t$ student para muestras pareadas de 5,354 y con intervalo de confianza del $95 \%$, diferencia significativa $(p<0,05)$ (Tabla 6).

El pH promedio hallado en los partos vaginales fue 7,24, con un mínimo de 7,12 y un máximo de 7,34 . La $\mathrm{pCO}_{2}$ promedio fue 40,49 , el $\mathrm{PO}_{2}$ promedio 25,79 y un exceso de base de $-1,4$, en promedio (Tabla 6).

Tabla 6. Características del neonato.

\begin{tabular}{lrr}
\hline & Promedio & \multicolumn{1}{c}{ DE } \\
\hline Ápgar a 1 minuto & & \\
Ápgar a 5 minutos & 9,4 & 0,72 \\
pH en sangre venosa umbilical & 7,24 & 0,13 \\
Peso promedio RN (g) & 2930,5 & 0,05 \\
& & 395,7
\end{tabular}




\section{DISCUSIÓN}

Hay que admitir que, el parto puede constituir para la mujer el más intenso dolor que experimentó jamás. La analgesia epidural por catéter requiere de gestantes que tengan un nivel de instrucción apropiado, para entender el método en uso y controlar su umbral de dolor $\left({ }^{1}\right)$.

Un $75 \%$ de nuestras gestantes había concluido su instrucción secundaria.

La Guia Nacional de Atención Integral de la Salud Reproductiva Ministerio de Salud, 2004, considera como mínimo que una gestante reciba 6 atenciones prenatales distribuidas a lo largo del embarazo y en nuestra investigación 43 pacientes $(71,7 \%)$ cumplieron con ello. La psicoprofilaxis obstétrica transfunde confianza y prepara psicosomáticamente a la gestante para 'un parto sin temor'. Ofrece más tranquilidad en el trabajo de parto $\left({ }^{1}\right)$. Nuestro estudio demostró que solo 7 gestantes -11,7\%- asistieron completamente a las sesiones de técnicas de psicoprofilaxis obstétrica.

Sobre los efectos en el dolor, en 1999, un estudio en que se empleó la misma escala de valoración del dolor confirmó que el bloqueo epidural durante el trabajo de parto logra un buen control del dolor $\left({ }^{13}\right)$. Durante la fase activa y expulsivo obtuvimos una analgesia satisfactoria y 33 parturientas requirieron de analgesia suplementaria, una sola vez; este grupo fue el más numeroso y utilizó $45 \mathrm{mg}$ de BPV al $0,25 \%$. Existe cierta controversia acerca de la conveniencia de continuar con el bolo epidural durante el expulsivo, dada la necesidad de mantener el bloqueo motor en su mínima expresión y permitir con ello mejorar la calidad de pujo de la parturienta; por ello, se le asocia a mayor frecuencia de partos vaginales instrumentales $\left({ }^{10}\right)$. En nuestro estudio, de las gestantes que recibieron una sola dosis, 22 presentaron fuerza adecuada en los músculos perineales, pero 5 pacien- tes, que recibieron analgesia suplementaria por segunda vez, presentaron cierta dificultad para pujar.

Una revisión hecha en el 2001, refiere que el bloqueo epidural segmentario con 6 $\mathrm{mL}$, a través del catéter, se distribuye segmentariamente desde la décima raíz dorsal a la $5^{\text {a }}$ sacra; el periné no se anestesia hasta el periodo expulsivo y es menos probable que se pueda afectar la dinámica uterina, disminuyendo a la vez la frecuencia de posiciones occípito posteriores y transversas, ya que la cabeza fetal puede rotar en el suelo pélvico; además, se produce menos hipotensión $\left({ }^{14}\right)$.

En contraste, al valorar el dermatoma sensitivo máximo alcanzado, encontramos un nivel alto para este procedimiento, T8 en $47,2 \%$ de los casos, lo cual puede explicarse por la introducción del catéter 5 $\mathrm{cm}$ en el espacio epidural y la dirección cefálica.

La hipotensión arterial es el efecto colateral más frecuente, que suele darse en $18 \%$ de los casos, siendo su morbilidad manifiesta si no es detectada $\left({ }^{14}\right)$. En nuestro estudio, se registró un caso, que fue tratado con venoclisis con solución de cloruro de sodio al 9 o/oo, $1000 \mathrm{~mL}$, desplazando el útero hacia la izquierda, no habiendo necesidad de emplear vasopresores.

La evaluación del monitoreo electrónico fetal intraparto posterior a la analgesia puede mostrar una variabilidad disminuida transitoria inicial, que se recupera posteriormente. Este hecho ha sido correlacionado con un estudio de gases arteriovenosos en sangre de cordón, al nacimiento, los cuales no han mostrado evidencia clínica compatible con depresión neonatal y acidosis. Igualmente, no se ha demostrado efectos desfavorables sobre el puntaje Ápgar o los parámetros de neurocomportamiento en el período neonatal inmediato $\left({ }^{15}\right)$. La variabilidad latido a latido se reduce después de la 
analgesia de parto. Con relación a las contracciones uterinas, éstas se incrementan en frecuencia después de la analgesia de parto $\left({ }^{16}\right)$. En nuestro estudio, la frecuencia cardiaca fetal disminuyó en promedio unos 12 latidos por minuto, postanalgesia (a los 20 minutos del procedimiento). Algunos estudios apoyan el hecho de una prolongación de la fase activa del parto en las pacientes sometidas a analgesia epidural. Sin embargo, en la población estudiada $\left({ }^{10}\right)$ se demuestra que la duración de la fase activa y del periodo expulsivo del parto fue significativamente menor y esta diferencia no se modificaba al considerar la paridad de las pacientes o cuando se consideraba la duración total del parto. Resaltamos el hecho de no haber encontrado prolongación del trabajo de parto en el primer y segundo periodo, comparado con la literatura examinada, dando un promedio de 2 horas con 42 minutos para la primípara y 1 hora 55 minutos para las multíparas Este acortamiento o reducción en el tiempo de duración de la fase activa podría ser explicada por varios factores, como son indicación y seguimiento continuo por parte del ginecoobstetra del monitoreo electrónico fetal.

La dilatación cervical promedio fue 5,25 $\mathrm{cm}$, encontrando a la parturienta en fase activa, con una incorporación cervical de $90 \%$ y la altura de descenso de presentación fetal en -2 .

El uso de oxitocina $(13,1 \mathrm{mU} / \mathrm{min}$, promedio), luego de la colocación de la analgesia en $88,3 \%$ de los casos, para la conducción del trabajo de parto, también es considerada como un factor importante. Se comprueba que existe un aumento del requerimiento de oxitocina en la conducción del parto en nulíparas con analgesia epidural, sugiriendo vigilancia en el progreso y uso agresivo de oxitocina, que podría ser factor fundamental en un número menor de cesáreas; por lo que, este medi- camento deberá ser utilizado con vigilancia estricta y uso racional $\left({ }^{9}\right)$.

La tasa de cesáreas de $11,2 \%$ (7 casos) podría haber estado influenciada por muchos factores, como los problemas médico legales. Esto se debe a que el binomio madre-niño debe ser considerado prioritariamente. Más aún, en mujeres con disfunción del parto, el dolor es mayor y por lo tanto solicitan analgesia de parto, requiriendo dosis suplementarias repetidas. Se requerirá una cesárea, independientemente de que la mujer hubiera recibido analgesia epidural o no.

Sobre los efectos en el neonato, el puntaje Ápgar y el pH de sangre venosa umbilical fueron normales. En nuestro estudio, ningún caso fue registrado como asfixia severa, con $\mathrm{pH}<6,99$. Un estudio dirigido a los neonatos comparó a los recién nacidos de parto normal y de madres que recibieron bloqueo de anestesia regional, experimentando estos últimos más hipoglicemia. Se necesita más ensayos para investigar estas posibilidades $\left({ }^{17}\right)$.

Hablando de complicaciones, la analgesia epidural en el trabajo de parto, a pesar de tener una relación beneficio / riesgo favorable, no debemos olvidar que es una técnica inocua. La inconveniencia del uso del catéter epidural, la incidencia de parestesias publicada -que varía entre 35 y $56 \%$-, así como la migración del catéter, rotura, anudamiento, restan eficacia a la técnica epidural de analgesia de parto $\left({ }^{18,19}\right)$.

Se presentó un caso anecdótico en nuestro estudio: Hubo dificultad para extraer el catéter epidural, el mismo que obligó a levantar a la paciente en decúbito dorsal, arquearla y retirar el catéter, ocasionando malestar a la puérpera.

En un metaanálisis, se encontró que el riesgo de punción accidental de la duramadre para el control del dolor de tra- 
bajo de parto fue $1,5 \%$ con intervalo de confianza de $95 \%\left({ }^{20-22}\right)$. En nuestro estudio, se presentó en un caso de punción dural, de un total de 60 parturientas, que fue tratado con hidratación endovenosa y reposo absoluto, sin almohada, el mismo que terminó en cesárea por el diagnostico asociado de mala actitud de presentación fetal. Sin embargo, resaltamos que fue el dolor lumbar presentado en el puerperio el que restó confort a dos pacientes.

\section{REFERENCIAS BIBLIOGRÁFICAS}

1. Celesia M.C. Rev. del Hospital Materno Infantil Ramón Sarda. 2003;22(1):28-31.

2. Mac Arthur C, Sheman A, May A. La analgesia epidural con bajas dosis disminuye el porcentaje de partos vaginales instrumentales sin comprometer la calidad de la analgesia. The Lancent. 2001;358;19-23.

3. Howell CS, Kidd C, Robert W, Upton P, Luckm L, Jones $\mathrm{P}$, et al. A randomised controlled trial of epidural, compared with non - epidural analgesia in labour. Brit J Obstet Ginecol.. 2001;108(1):27-33.

4. Elton CD, Ali P, Mushambi MC. Walking extradurals in labour step forward? Brit J Anaesthesia. 1997;79(5):551-4.

5. Morgan BM. Walking epidurals in labour. Anaesthesia. 1995:50:839-40.

6. Pun R. Levobupivacaina $0,25 \%$ más fentanilo en analgesia epidural de parto. Estudio prospectivo del HNGAI (Tesis de Especialista) Lima, Perú. Facultad de Medicina UNMSM. 2003: $14 \mathrm{p}$

7. Nystedt A, Edvardsson D, Willman A. Epidural analgesia for pain relief in labor and childbirth a review with a systematic approach. Blackwell Publishing. J of Clinical. 2004;13:45566.

8. Jodi S, Dashe, MD, Beverly B. Rogers, MD, Donald D. Mcintire, $\mathrm{PhD}$. Epidural analgesia and intrapartum fever: placental findings. Obstet Gynecol. 1999;93:341-4.

9. Decca L, Dadlos C, Fratelli N. La analgesia epidural no genera mayor cantidad de cesáreas. J Maternal Fetal Neonatal Med. 2004; 16 (2):115-8.

10. Santos J, Ruano A, Beltrán, et al. Efecto de la analgesia epidural sobre la duración y tipo de parto. Clin Invest Ginecol Obstet. 2004;31 (2):36-43.
11. Isaza G. La Práctica del Método Psicoprofiláctico para el parto sin dolor en Medellín, Colombia. Rev Colomb Obstet Ginecol. 1959;10:226.

12. Guía Nacional de Atención Integral de la Salud Sexual y Reproductiva MINSA 2004.

13. Sánchez MC, Uribarri FJ. Analgesia epidural para el trabajo de parto. Rev Soc Esp Dolor. 1999;6:406-11.

14. Becerro C, Becerro G. Analgesia y anestesia en obstetricia. Toko-Gin Pract. 2001;60(2):80-94.

15. Eleva N. Neonatal monitoring after maternal analgesia in labor. J Clin Monitoring. 2001;16:597-608.

16. Maldonado E. Repercusiones de la Analgesia de Parto en el Monitoreo Electrónico Fetal y pH. IEMP (Tesis de Especialista) Lima, Peru. UNMSM Fac. Medicina. 2006: 40p

17. Swanstrom S, Bratteby LE. Metabolic effects of obstetric regional analgesia and of asphyxia in the newborn infant during the first two hours alter birth. I. Arterial blood glucose concentrations. Acta Pediatr Escandinava. 2001:791-800.

18. Bishton JM, Martin PH, Vermon JM, Liu WH. Factors Influencing epidural Catéter migration. Anaesthesia. 1992;47(7):610-2.

19. Wlody D. Complications of regional anesthesia in obstetrics. Clin Obstet Gynecol. 2003;46:667-8.

20. Choi PT, Galinski SE, Takeuchi L, Lucas S, Tamayo C, Jadad AR. PPOH Is a common complication of neuraxial blockade in parturients: a meta-analysis of obstetrical studies. Can J Anaesth. 2003;50:460-9.

21. Phillips SM, Stedeford SC, Hartsilver E, et al. Epidural abscess complicating insertion of epidural ca theters. Br S Anaesthesia. 2002;89:778-2.

22. Zakowski M. Complications associated with regional anesthesia in the obstetric pacient. Semin Perinatol. 2002:26:154-68.

Manuscrito recibido el 01 de setiembre de 2006 y aceptado para publicación el 29 setiembre de 2006.

Correspondencia:

Silvia Erlinda Arias Lazarte

Av Brasil 3377 Dpto: 602 Magdalena del Mar

Tlf. 4623741

Correo-e:erlindarias@hotmail.com 